\title{
Observation of a crossover from nodal to gapped superconductivity in $\operatorname{Lu}_{x} \mathrm{Zr}_{1-x} B_{12}$
}

\author{
Franziska K. K. Kirschner, ${ }^{1, *}$ Nikolay E. Sluchanko, ${ }^{2,3}$ Vladimir B. Filipov, ${ }^{4}$ Francis L. Pratt, ${ }^{5}$ Chris Baines, ${ }^{6}$ \\ Natalya Yu. Shitsevalova, ${ }^{4}$ and Stephen J. Blundell ${ }^{1, \dagger}$ \\ ${ }^{1}$ Department of Physics, University of Oxford, Clarendon Laboratory, Parks Road, Oxford OX1 3PU, United Kingdom \\ ${ }^{2}$ A. M. Prokhorov General Physics Institute of RAS, 38 Vavilov Street, 119991 Moscow, Russia \\ ${ }^{3}$ Moscow Institute of Physics and Technology, Institutskii per. 9, Dolgoprudnyi, Moscow Region 141700, Russia \\ ${ }^{4}$ Frantsevich Institute for Problems of Materials Science NAS, 3, Krzhyzhanovsky str., Kiev 03680, Ukraine \\ ${ }^{5}$ ISIS Facility, STFC Rutherford Appleton Laboratory, Chilton, Didcot, Oxfordshire OX11 OQX, United Kingdom \\ ${ }^{6}$ Laboratory for Muon Spin Spectroscopy, Paul Scherrer Institute, CH-5232 Villigen, PSI, Switzerland
}

(Received 16 April 2018; revised manuscript received 2 August 2018; published 5 September 2018)

\begin{abstract}
We have determined the superconducting and magnetic properties of four samples of $\operatorname{Lu}_{x} \mathrm{Zr}_{1-x} \mathrm{~B}_{12}(x=0.04$, $0.07,0.17$, and 0.8$)$ using muon spin rotation $(\mu \mathrm{SR})$ and magnetometry measurements. We observed a strong magnetic signal in both the $\mu \mathrm{SR}$ and magnetometry data in one sample $(x=0.07)$, likely caused by the formation of static moments of size $\approx 1 \mu_{\mathrm{B}}$ due to a clustering effect of the $\mathrm{Lu}^{3+}$ ions. In all other samples, we find only a small magnetic signal in the $\mu \mathrm{SR}$ data thought to originate from boron nuclei in the $\mathrm{B}_{12}$ cages. The superconductivity is found to evolve with $x$, with a decrease in $x$ resulting in an increase in critical temperature and a decrease of the penetration depth. Most remarkably, we find evidence for the formation of nodes in the superconducting gap for $x \leqslant 0.17$, providing a potential new example of an $s$-to-s $+d$-wave crossover in a superconductor.
\end{abstract}

DOI: 10.1103/PhysRevB.98.094505

Introduction. Since the discovery of superconductivity in $\mathrm{MgB}_{2}$ [1], there has been great interest in searching for superconductivity in a wide class of borides. $\mathrm{ZrB}_{12}$ is a $\mathrm{BCS}$ superconductor with one of the highest critical temperatures $\left(T_{\mathrm{c}}=6 \mathrm{~K}\right)$ among boron-rich compounds [2]. $\mathrm{LuB}_{12}$, another dodecaboride, is also a superconductor, albeit with a much lower $T_{\mathrm{c}}=0.48 \mathrm{~K}$ [2] despite having a very similar crystal structure [3,4], electronic density of states [5-7], and phonon density of states $[8,9]$ to $\mathrm{ZrB}_{12}$. It is thought that the high $T_{\mathrm{c}}$ in $\mathrm{ZrB}_{12}$ originates from the soft vibrations of $\mathrm{Zr}^{4+}$ ions in the boron cages (this structure is shown in Fig. 1). These vibrations lead to a strong electron-phonon interaction [10-12]. In $\mathrm{LuB}_{12}$, vibrations of $\mathrm{Lu}^{3+}$ ions have almost no contribution to the electron-phonon coupling, perhaps due to the 'volume filling factor' of the B cages, which tunes the hybridization between the $\mathrm{Lu} / \mathrm{Zr}$ and B orbitals [9]. Another factor which may contribute to the lower $T_{\mathrm{c}}$ in $\mathrm{LuB}_{12}$ is the development of an electron instability due to the formation of dynamic charge stripes [13]. Though $\mathrm{LuB}_{12}$ appears to display $s$-wave behavior [14], there has been considerable debate surrounding the nature of the superconducting gap function in $\mathrm{ZrB}_{12}$. It has been suggested that $\mathrm{ZrB}_{12}$ is either a single-gap $s$-wave [15,16], two-gap $s$-wave [17], or a $d$-wave [10] superconductor, with its Fermi surface composed of one open and two closed sheets [5,7].

Nonmagnetic impurity substitutions impact on superconducting properties in various ways dependent on the pairbreaking mechanism. For example, Anderson's theorem

\footnotetext{
*franziska.kirschner@physics.ox.ac.uk

${ }^{\dagger}$ stephen.blundell@physics.ox.ac.uk
}

implies that a small number of nonmagnetic impurities can dramatically suppress superconductivity in the case of an anisotropic gap in a $d$-wave superconductor [18-20]. Experiments on cuprates reveal that a spinless impurity introduced into the high-temperature superconductor host produces a large and spatially extended alternating magnetic polarization in its vicinity [21]. Somewhat analogous behavior was found in $\mathrm{Lu}_{x} \mathrm{Zr}_{1-x} \mathrm{~B}_{12}$, in which nonmagnetic $\mathrm{Lu}^{3+}$ ions are substituted for $\mathrm{Zr}^{4+}$ ions. Spin-polarized nanodomains of size $\approx 5 \AA$, containing moments $\approx 6 \mu_{\mathrm{B}}$ and nucleated around the $\mathrm{Lu}^{3+}$ ions, were found in some crystals [22] but not in others [23]. This is possibly due to details of the distribution of the $\mathrm{Lu}^{3+}$ ions in the lattice or the presence of vacancies (as found in $\mathrm{YB}_{6}[24]$ ).

In this paper, we report muon spin rotation $(\mu \mathrm{SR})$ and magnetometry experiments on four samples from the $\mathrm{Lu}_{x} \mathrm{Zr}_{1-x} \mathrm{~B}_{12}$ family of superconductors to determine their superconducting and magnetic properties. We focus on samples relatively close to the stoichiometric $\mathrm{LuB}_{12}$ and $\mathrm{ZrB}_{12}$ end members of this family of compounds, in order to investigate the effect of nonmagnetic substitutions in the low-doping $(\leqslant 20 \%$ substitution, or $x \leqslant 0.2$ and $x \geqslant 0.8)$ regime. One sample showed evidence of magnetism from the nanodomains postulated in Ref. [22]. Remarkably, we find that the increase in $T_{\mathrm{c}}$ with decreasing $x$ is accompanied by the formation of nodes in the superconducting gap. We also find that, while the magnetism is sample dependent, the superconducting properties are nevertheless robust.

Experimental details. Four single crystals of $\mathrm{Lu}_{x} \mathrm{Zr}_{1-x} \mathrm{~B}_{12}$ were investigated in this experiment; these included one 'magnetic' sample, which had previously displayed the nanodomain behavior, and three 'nonmagnetic' samples, which 


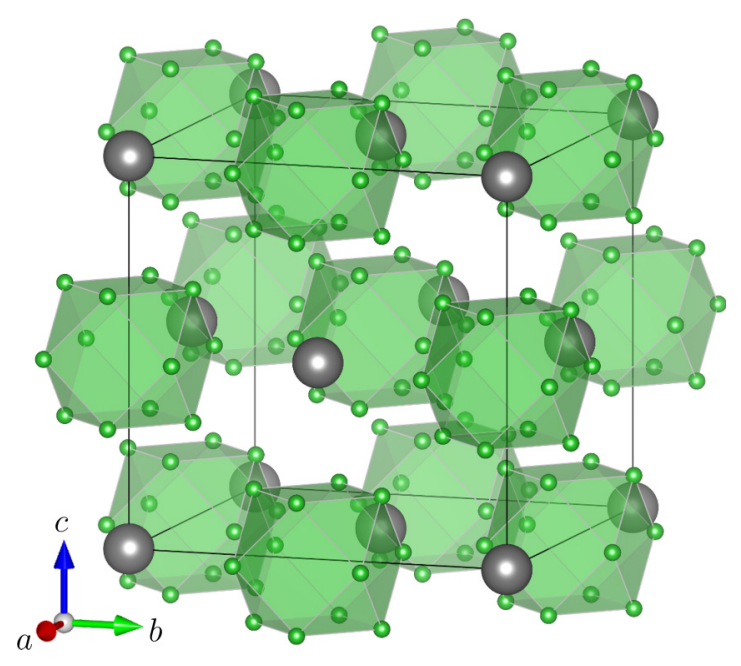

FIG. 1. The unit cell of $R \mathrm{~B}_{12}(R=\mathrm{Lu}$ or $\mathrm{Zr})$. The rare earth $(R)$ atoms are gray, and the $\mathrm{B}$ atoms are green. The $\mathrm{B}_{12}$ cages are shown in green.

displayed no such phenomena. The 'magnetic' sample, $x=0.07$, was identical to that used in Ref. [22]. The remaining three samples, $x=0.04,0.17$, and 0.8 , were 'nonmagnetic' and identical to those studied in Ref. [23]. Details of the crystal growth are described in Refs. [22] and [23].

The crystal growth methods were identical for both the magnetic and nonmagnetic samples, with the single crystal samples being grown from a sintered rod. However, the sintered rods were obtained via different methods. For the magnetic sample, the sintered rod was produced from a powder of the crushed single crystal $\mathrm{LuB}_{12}$ and $\mathrm{ZrB}_{12}$. Both powders were mixed and sintered into rods at $1750{ }^{\circ} \mathrm{C}$ in a vacuum; this method is unable to guarantee a homogeneous mixture of $\mathrm{Lu}$ and $\mathrm{Zr}$. Since there was a gradual re-melting of the rod during the synthesis of the single crystal, we cannot exclude an inhomogeneous distribution of $\mathrm{Lu}$ and $\mathrm{Zr}$ in the final crystal. In the case of the nonmagnetic samples, amorphous boron was mixed with $\mathrm{Lu}$ and $\mathrm{Zr}$ metal oxides $\left(\mathrm{Lu}_{2} \mathrm{O}_{3}\right.$ and $\left.\mathrm{ZrO}_{2}\right)$. These mixtures were pressed into tablets and held in a vacuum furnace for two hours at $1650^{\circ} \mathrm{C}$ to perform a solid-state synthesis, producing $(\mathrm{Lu}, \mathrm{Zr}) \mathrm{B}_{12}$. To achieve a homogeneous distribution of the metal components after synthesis the tablets were crushed, pressed, and annealed at $1650{ }^{\circ} \mathrm{C}$ in vacuum. Then they were crushed and pressed once more to produce the rods, which were sintered in vacuum at $1750{ }^{\circ} \mathrm{C}$ for one hour. As this method for producing the sintered rods had one more homogenizing annealing, we anticipate the $\mathrm{Lu} / \mathrm{Zr}$ distribution in these samples to be significantly more homogeneous than in the sample produced using the first synthesis method.

$\mu$ SR experiments $[25,26]$ were performed using a dilution refrigerator and ${ }^{3} \mathrm{He}$ sorption cryostat mounted on the MuSR spectrometer at the ISIS pulsed muon facility (Rutherford Appleton Laboratory, UK) [27]. Further experiments were carried out using the low temperature facility (LTF) and general purpose spectrometer (GPS) at the Swiss Muon Source. Transverse-field (TF) measurements were made to probe the superconducting ground state and its evolution with $x$. Zerofield (ZF) and longitudinal-field (LF) measurements were carried out in order to test for magnetic phases in the sample.
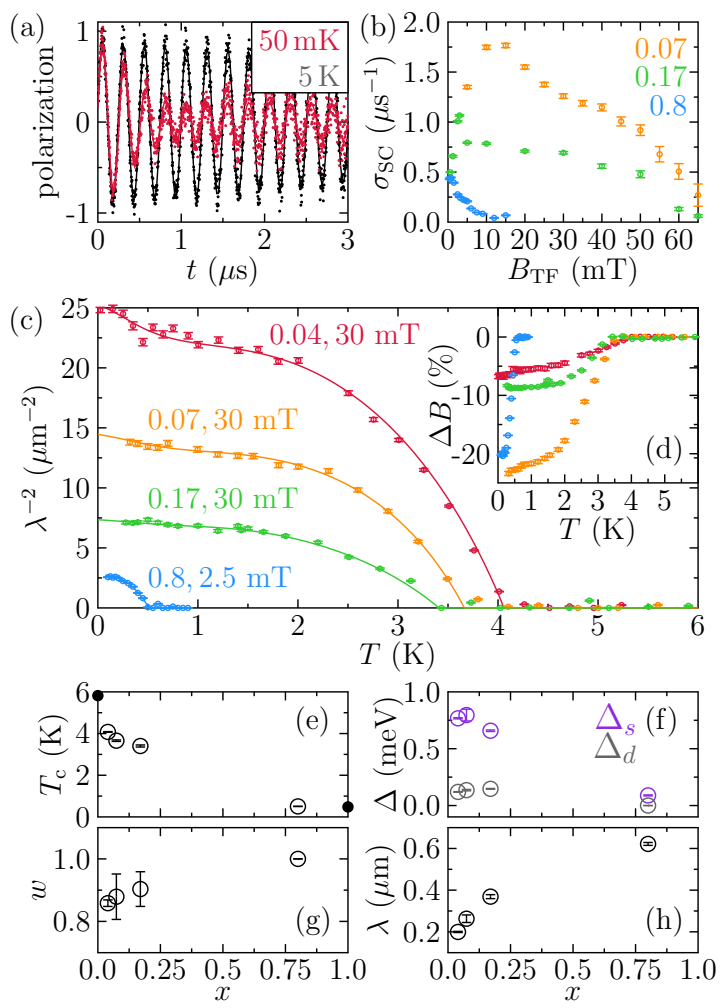

FIG. 2. (a) Sample TF- $\mu$ SR spectra above and below $T_{\mathrm{c}}$ for $x=0.04$. Fits as in Eq. (1) are also plotted. (b) Field dependence of the relaxation due to superconductivity for $x=0.07$ at $1.5 \mathrm{~K}$ (orange), $x=0.17$ at $1.5 \mathrm{~K}$ (green), and $x=0.8$ at $0.1 \mathrm{~K}$ (blue). (c) Temperature dependence of the inverse square penetration depth, with $s+d$-wave fits for $x=0.04,0.07$, and $x=0.17$, and an $s$-wave fit for $x=0.8$. (d) $T$ dependence of the field shift $\Delta B=B_{\mathrm{SC}}-B_{\mathrm{TF}}$, expressed as a percentage of $B_{\mathrm{TF}}$, due to superconductivity. The evolution of $T_{\mathrm{c}}$ (the zero-field value, extrapolated from magnetometry data), the superconducting gaps, the gap ratio, and the penetration depth with $x$ are given in (e), (f), (g) and (h), respectively. The unfilled black points in (e) are the $T_{\mathrm{c}}$ values for $\mathrm{ZrB}_{12}$ and $\mathrm{LuB}_{12}$ from Ref. [2], and the triangles are recently measured points for intermediate doping [30].

All of the $\mu$ SR data were analyzed using WiMDA [28]. Magnetometry measurements were carried out on a Quantum Design SQUID magnetometer.

Superconductivity. TF- $\mu \mathrm{SR}$ measurements were performed on all of the samples to determine their superconducting properties. $x=0.04,0.07$, and 0.17 were measured above and below $T_{\mathrm{c}}$ in transverse applied fields $B_{\mathrm{TF}}$ of $30 \mathrm{mT}$ and $x=0.8$ was measured in $B_{\mathrm{TF}}=2.5 \mathrm{mT}$ (fields were chosen in order to lie below $B_{\mathrm{c} 2}$ for each compound; see the Supplemental Material [29] for $B_{\mathrm{c} 2}$ values). Measurements were taken in field-cooled mode, where $B_{\mathrm{TF}}$ is applied above $T_{\mathrm{c}}$ before the sample is cooled down. A representative spectrum for $x=0.04$ is shown in Fig. 2(a). Transverse field sweeps were also made to determine the field dependence of the internal field distribution of the superconducting state.

The data were fitted with

$$
\begin{aligned}
A= & A_{\mathrm{TF}} \cos \left(\gamma_{\mu} B_{\mathrm{TF}} t+\phi\right) e^{-\frac{\sigma_{\mathrm{TF}}^{2} t}{2}} \\
& +A_{\mathrm{SC}} \cos \left(\gamma_{\mu} B_{\mathrm{SC}} t+\phi\right) e^{-\frac{\sigma^{2} t}{2}},
\end{aligned}
$$


where $\gamma_{\mu}=2 \pi \times 135.5 \mathrm{MHz} \mathrm{T}^{-1}$ is the gyromagnetic ratio of the muon and $\phi$ is related to the detector geometry (the data were divided among eight groups of detectors with $\phi$ fitted for each group). The first term corresponds to a background signal arising from muons that stop outside of the superconducting volume: in the cryostat, sample holder, and nonsuperconducting regions of the sample. These muons precess only in the external field and experience a small Gaussian relaxation. The second term corresponds to muons in the superconductor, which experience an approximately Gaussian relaxation arising from the field distribution of the vortex lattice. These muons also experience a small, temperature independent relaxation from nuclear moments, giving a total relaxation $\sigma(T)=\sqrt{\sigma_{\mathrm{SC}}^{2}(T)+\sigma_{\text {nucl }}^{2}}$.

The transverse field dependences of $\sigma_{\mathrm{SC}}$ for $x=0.07$, 0.17 , and 0.8 are shown in Fig. 2(b). There is a muon quadrupolar resonance due to the boron nuclei at $\approx 4 \mathrm{mT}$, which may affect the low-field dependence (this will be further discussed below). Apart from $x=0.8$, the upper critical fields $B_{\mathrm{c} 2}$ of all the samples are larger than the fields resolvable by the experiment; these have instead been calculated from magnetometry and heat capacity measurements in Refs. [22] and [23], and are shown in Ref. [29].

Assuming all of the samples are type II superconductors with an isotropic hexagonal Abrikosov vortex lattice in the $a-b$ plane that can be described by Ginzburg-Landau theory, the in-plane penetration depth $\lambda$ can be extracted from the relaxation due to superconductivity using [31] $\sigma_{\mathrm{SC}}=0.0609 \gamma_{\mu} \phi_{0} \lambda^{-2}(T)$, where $\phi_{0}=2.069 \times 10^{-15} \mathrm{~Wb}$ is the magnetic flux quantum. This formula is an approximation which holds for $0.13 / \kappa^{2} \ll B_{\mathrm{TF}} / B_{\mathrm{c} 2} \ll 1$ where $\kappa$ is the Ginzburg-Landau parameter [29]. By comparing this approximation to the analytical relationship between $\sigma_{\mathrm{SC}}$ and the applied field in Ref. [31], we find it correctly describes this relationship to within $10 \%$ for the $x=0.04$ and 0.07 samples, and to within $20 \%$ for $x=0.17$ and 0.8 . The temperature dependence of $\lambda^{-2}$ is shown in Fig. 2(c), and the corresponding field shifts due to the vortex lattice $\Delta B=B_{\mathrm{SC}}-B_{\mathrm{TF}}$ are given in Fig. 2(d).

To determine the nature of the superconducting gaps in the samples, we fitted the data with single-gap BCS $s$-wave and $d$-wave models, as well as two-gap $s+s$ - and $s+d$-wave models. The BCS model of the normalized superfluid density of a superconductor is given by [32]:

$$
\begin{aligned}
\tilde{n}_{\mathrm{s}}(T) & =\frac{\lambda^{-2}(T)}{\lambda^{-2}(0)} \\
& =1+\frac{1}{\pi} \int_{0}^{2 \pi} \int_{\Delta(\phi, T)}^{\infty} \frac{\partial f}{\partial E} \frac{E \mathrm{~d} E \mathrm{~d} \phi}{\sqrt{E^{2}-\Delta^{2}(\phi, T)}},
\end{aligned}
$$

where $\Delta(\phi, T)$ is the superconducting gap function, and $f=\left[1+\exp \left(E / k_{\mathrm{B}} T\right)\right]^{-1}$ is the Fermi function. The gap function can be approximated as $\Delta(\phi, T)=$ $\Delta(\phi) \tanh \left(1.82\left[1.018\left(T_{\mathrm{c}} / T-1\right)\right]^{0.51}\right)$. The angular gap function $\Delta(\phi)=\Delta_{0}$ for $s$-wave superconductors and $\Delta(\phi)=\Delta_{0} \cos (2 \phi)$ for $d$-wave (nodal) superconductors. For multigap models, the total $\tilde{n}_{\mathrm{s}}(T)$ is given by a weighted sum of the superfluid densities for the individual gaps:
TABLE I. The values of $\chi^{2}$ for the best fits to various single and multigap models, using Eq. (2) for all of the samples (details of the fits are given in the main text.). The values in bold indicate the best fitting models.

\begin{tabular}{lcccc}
\hline \hline$x$ & $s$ wave & $d$ wave & $s+s$ wave & $s+d$ wave \\
\hline 0.04 & 20.9 & 23.2 & 15.8 & $\mathbf{1 4 . 1}$ \\
0.07 & 11.9 & 10.9 & 12.1 & $\mathbf{1 0 . 0}$ \\
0.17 & 18.8 & 16.5 & 16.6 & $\mathbf{1 3 . 8}$ \\
0.8 & $\mathbf{1 6 . 9}$ & 19.7 & 19.3 & 22.6 \\
\hline \hline
\end{tabular}

$\tilde{n}_{\mathrm{s}}(T)=w \tilde{n}_{\mathrm{s}}^{\text {gap } 1}(T)+(1-w) \tilde{n}_{\mathrm{s}}^{\text {gap } 2}(T)$. In the case of the $s+d$-wave fits, the first gap is $s$ wave, whereas the second gap is $d$ wave.

We find that $x=0.04,0.07$, and 0.17 are $s+d$-wave superconductors, whereas $x=0.8$ is purely $s$ wave. $\chi^{2}$ values for $s-, d-, s+s$-, and $s+d$-wave fits for all of the samples are shown in Table I. A key indicator of nodal superconductivity is the linear dependence of $\lambda^{-2}$ at low $T$, which is observed in $x=0.04,0.07$, and 0.17. $x=0.8$, on the other hand, shows a low temperature plateau in $\lambda^{-2}$, corresponding to a fully gapped superconductor where low-energy excitations are strongly suppressed [33]. This is consistent with the $s$-wave superconductivity observed in $\mathrm{LuB}_{12}$ [14]. Further discussion of this fitting can be found in the Supplemental Material [29]. Our observations show that the nodal gap becomes less anisotropic and eventually becomes isotropic as $x$ increases. This change of gap structure is accompanied by a rapid suppression of $T_{\mathrm{c}}$, shown in Fig. 2(e). The fitted values of the $s$ - and $d$-wave superconducting gaps, $\Delta_{s}$ and $\Delta_{d}$, respectively, are shown in Fig. 2(f); we find that along with the complete suppression of the nodal gap [indicated by $w \rightarrow 1$ in Fig. 2(g)], the $s$-wave gap also decreases significantly in size with increasing $x$. For all of the samples, we find that the gap to $T_{\mathrm{c}}$ values $\Delta_{s} / k_{\mathrm{B}} T_{\mathrm{c}} \approx 2-2.5$ (>1.76, the BCS value) for the larger $s$-wave gap, meaning these compounds are likely strongly-coupled superconductors (although for the multigapped samples, $\Delta_{d} / k_{\mathrm{B}} T_{\mathrm{c}} \approx 0.3-0.5$ in all cases). As $x$ increases, the superfluid density $\left[n_{\mathrm{s}} \propto \lambda^{-2}(0)\right]$ is suppressed, leading to a longer penetration depth as shown in Fig. 2(h). This agrees well with the two peaks observed in the field dependence of $\sigma_{\mathrm{SC}}$ for $x=0.07$ [see Fig. 2(b)]. The peak at $B_{\mathrm{TF}} \approx 12 \mathrm{mT}$ may be a reflection of the field dependence of the weak $d$-wave term on top of the $s$-wave term. As the $d$-wave contribution is more easily suppressed by an external field, we expect $w$ to be field dependent. From the amplitude of the $12 \mathrm{mT}$ peak we can estimate that the corrected value of $w$ in the low field London limit should be closer to 0.7 for this sample and will be similarly reduced for the other low- $x$ samples.

$\mathrm{Zr}\left([\mathrm{Kr}] 5 s^{2} 4 d^{2}\right)$ contributes two $d$ electrons to the conduction band whereas $\mathrm{Lu}\left([\mathrm{Xe}] 4 f^{14} 6 s^{2} 5 d^{1}\right)$ only contributes one. This results in increased $d$-wave orbital pairing and favors nodal superconductivity. The observed gap evolution in the $\mathrm{Lu}_{x} \mathrm{Zr}_{1-x} \mathrm{~B}_{12}$ is similar to that seen in Fe-based superconductors. The $\mathrm{Ba}_{1-x} \mathrm{~K}_{x} \mathrm{Fe}_{2} \mathrm{As}_{2}$ family of materials shows fully gapped behavior for $x=0.4$, with line nodes appearing at $x=1$ [34]. A similar transition is 

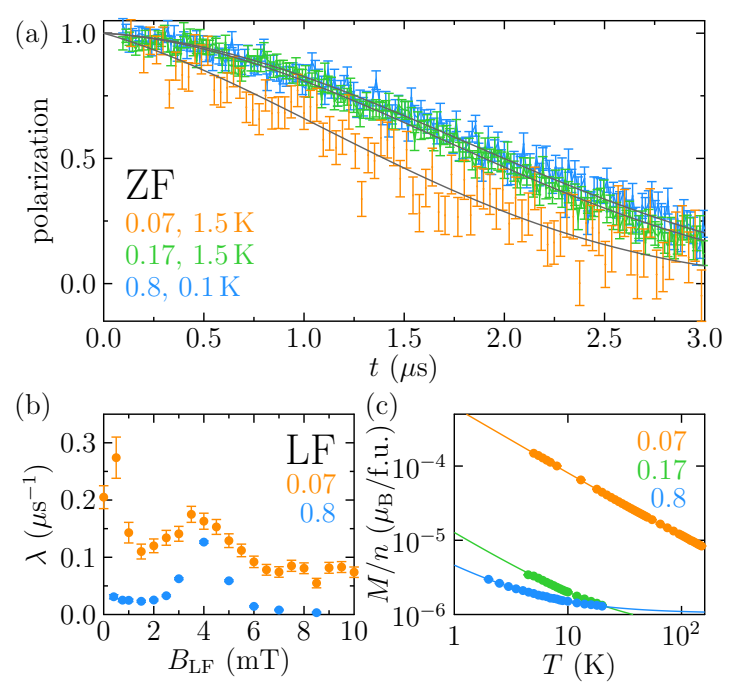

FIG. 3. (a) Sample ZF- $\mu$ SR spectra in the superconducting state. (b) The field dependence of the relaxation for one 'magnetic' sample ( $x=0.07$, orange) and one 'nonmagnetic' sample ( $x=0.8$, blue). The measurements for $x=0.07$ were taken at $5 \mathrm{~K}$ and for $x=0.8$ at $0.7 \mathrm{~K}$. (c) The temperature dependence of the magnetization per formula unit.

achieved in $\mathrm{Ba}_{0.65} \mathrm{Rb}_{0.35} \mathrm{Fe}_{2} \mathrm{As}_{2}$ using hydrostatic pressure, which promotes a nodal gap [35].

Magnetism. To address the apparent discrepancy between the differing magnetic behaviours of this family of compounds observed in Refs. [22] and [23], ZF- and LF- $\mu$ SR experiments were carried out. ZF spectra below $T_{\mathrm{c}}$ for one 'magnetic' sample $(x=0.07)$ and two 'nonmagnetic' samples $(x=0.17$ and 0.8$)$ are shown in Fig. 3(a). For all samples there was no detectable change in the ZF spectrum as $T$ was increased above $T_{\mathrm{c}}$, meaning that any magnetism present in the samples does not compete with the superconducting phase. No oscillations were seen in the forward-backward asymmetry spectra, and there were also no discontinuous jumps in either the initial or the baseline asymmetry, which suggests there is no long-range order inside the sample. The data were fitted with the zero-field Kubo-Toyabe function [36] with an additional Lorentzian relaxation:

$$
A=A_{0}\left(\frac{1}{3}+\frac{2}{3}\left(1-\Delta_{\mathrm{ZF}}^{2} t^{2}\right) e^{-\Delta_{\mathrm{ZF}}^{2} t^{2} / 2}\right) e^{-\lambda_{\mathrm{ZF}} t}+A_{\mathrm{b}},
$$

where $A_{0}$ and $A_{\mathrm{b}}$ are the relaxing and baseline amplitudes. The relaxing amplitude corresponds to muon stopping in the magnetic portion of the sample, while the baseline amplitude represents muons landing in nonmagnetic regions, for example in the cryostat or sample holder. The Kubo-Toyabe function is often used to describe a system of static spins with a Gaussian field distribution characterized by an RMS width $\Delta_{\mathrm{ZF}} / \gamma_{\mu}$.

$\Delta_{\mathrm{ZF}}$ was found to be constant and temperature independent $\left[0.40(3) \mu \mathrm{s}^{-1}\right]$ across all of the samples. The additional Lorentzian relaxation was equal and very small for the two nonmagnetic samples $\left(\approx 0.05 \mu \mathrm{s}^{-1}\right)$ and significantly larger for the magnetic sample $\left(\approx 0.2 \mu \mathrm{s}^{-1}\right)$.

To further probe the nature of the magnetism, LF- $\mu$ SR was carried out on one magnetic and one nonmagnetic sample: $x=0.07$ and $x=0.8$, respectively. The data were fitted with a longitudinal-field Kubo-Toyabe function [37] with an additional Lorentzian relaxation $e^{-\lambda_{\text {ZF }} t}$; the field dependence of $\lambda_{\mathrm{ZF}}$ is plotted in Fig. 3(b). Both samples have a peak in their relaxation at $B \approx 4 \mathrm{mT}$, corresponding to the muon experiencing a quadrupolar resonance with the ${ }^{11} \mathrm{~B}$ nucleus $[38,39]$. The additional relaxation seen in the $\mathrm{ZF}$ spectrum of the magnetic sample is quenched above $\approx 1 \mathrm{mT}$, indicating an internal field on the order of $0.1 \mathrm{mT}$ arising from static magnetic moments (an applied longitudinal field $B_{\mathrm{LF}}>10 B_{\text {internal }}$ rapidly quenches relaxation arising from static moments [26,37]).

We have used density functional theory to determine the muon site in the unit cell. By mapping the electrostatic potential inside the unit cell, an approximate site can be obtained by examining the maxima of this potential. A potential maximum corresponds to a low energy associated with introducing a positive charge (for example the muon) and has been found to be a good approximation to more detailed relaxation calculations $[40,41]$. Our calculations imply the muon site is at $(0.5,0.5,0.5)$ in the unit cell, in the center of the $\mathrm{B}_{12}$ cage.

The Lorentzian relaxation in Eq. (3) likely arises due to static, dilute moments [42], and hence the relatively large $\lambda_{\mathrm{ZF}}$ for the magnetic sample may be due to the formation of dilute nanodomains consisting of static moments associated with the $\mathrm{Lu}$ atoms. The value of $\lambda_{\mathrm{ZF}}$, combined with the LF required to suppress the relaxation, provide an approximate RMS field width of $\approx 0.1 \mathrm{mT}$ associated with the Lorentzian field distribution produced by the dilute moments. Using dipolar field simulations, we can calculate the expected dipolar field at the muon site $r_{\mu}$. The field from moments $\mathbf{m}_{i}$ and positions $\mathbf{r}_{i}$ can be found via

$$
\mathbf{B}_{\text {dip }}=\frac{\mu_{0}}{4 \pi} \sum_{i} \frac{3\left(\mathbf{m}_{\mathbf{i}} \cdot \hat{\mathbf{r}}_{i \mu}\right) \hat{\mathbf{r}}_{i \mu}-\mathbf{m}_{\mathbf{i}}}{\left|\mathbf{r}_{i \mu}\right|^{3}},
$$

where $\hat{\mathbf{r}}_{i \mu}$ is the normalized vector between the muon and the moment $\mathbf{m}_{i}$. Using Eq. (4), we find domains of size $\sim 7 \AA$ give $\lambda_{\mathrm{ZF}} / \gamma_{\mu} \approx 0.1 \mathrm{mT}$, where the moments associated with these domains is $\approx 0.8 \mu_{\mathrm{B}}$.

The observed differences in magnetism are supported by bulk magnetometry measurements of $x=0.07,0.17$, and 0.8 , as shown in Fig. 3(c). The data were fitted to a Curie-Weiss function with an additional constant background magnetization:

$$
M=M_{0}+n \mu_{0} \mu^{2} H / 3 k_{\mathrm{B}} T,
$$

where $\mu$ is the effective magnetic moment per formula unit, $n$ is the number density, and $\mu_{0} H=2 \mathrm{mT}$ is the applied external field. We find that the moment per formula unit in the magnetic sample $\left(\mu_{0.07} \approx 1.18 \mu_{\mathrm{B}}\right.$; close to the moment size obtained from the dipole field calculations above) is significantly higher than for the nonmagnetic samples $\left(\mu_{0.17} \approx 0.2 \mu_{\mathrm{B}}, \mu_{0.8} \approx 0.1 \mu_{\mathrm{B}}\right)$.

From these data, we postulate that the ZF signal observed in the nonmagnetic samples, which is insensitive to $\mathrm{Lu}^{3+}$ concentration, is dominated by B nuclei in the cages, similar to that observed in $\mathrm{LuB}_{12}$ [43]. In addition to this nuclear signal, the magnetic sample also contains static, disordered moments, which may potentially be associated with clusters of $\mathrm{Lu}^{3+}$, similar to the phenomena discussed in Refs. [22] 
and [24]. As the apparent clustering effect is only present in one sample, we conclude that the differences in magnetism we observe are due to the detailed distribution of cations that is established during sample preparation, rather than an intrinsic effect linked to $x$.

Conclusion. In summary, we have carried out $\mu \mathrm{SR}$ and magnetometry measurements to probe the nature of the magnetism and superconductivity in the $\mathrm{Lu}_{x} \mathrm{Zr}_{1-x} \mathrm{~B}_{12}$ family of superconductors. ZF- $\mu$ SR, LF- $\mu$ SR, and magnetometry measurements reveal a strong magnetic signal in one sample, which was thought to contain magnetic nanodomains, compared to those samples with no domains. We attribute this sample dependence to a clustering effect in which the distribution of $\mathrm{Lu}^{3+}$ may affect the formation of static moments. We find that the moments associated with this effect are of order $1 \mu_{\mathrm{B}}$ per formula unit. Scanning tunneling microscopy or scanning electron microscopy may provide further insights into this effect. TF- $\mu$ SR measurements revealed that the superconductivity was robust to these variations in magnetism. We find that the increase in $T_{\mathrm{c}}$ associated with the decrease of $x$ is also accompanied by a decrease in penetration depth and the likely formation of nodes in the superconducting gap function. There are many extensions to the models we have used to fit our data. While our minimal models fit the data well, we stress that more in-depth studies of this family of materials are required to further probe the gap symmetries observed in our $\mu \mathrm{SR}$ data. The nodal superconductivity appears to occur even in the magnetic sample; we can deduce that the nodal gaps we observe are probably due to $d$-wave pairing, rather than $s$-wave pairing with accidental nodes: Impurity scattering (which is possible due to the variability in the magnetic properties of the sample) would broaden symmetry nodes and lift any accidental nodes, therefore removing the residual linear term in $\lambda^{-2}$. The unusual transition from nodal to gapped superconductivity is similar to that observed in iron pnictide superconductors, although we note that in the case of the pnictides, the formation of nodes in the superconducting gap is accompanied by a decrease in $T_{\mathrm{c}}$ and a decrease in the superfluid density [34,35]. Further study of the $\mathrm{Lu}_{x} \mathrm{Zr}_{1-x} \mathrm{~B}_{12}$ family of compounds may provide additional insights into the mechanisms behind nodal-togapped crossovers in high temperature superconductors. It has recently become possible to synthesize samples of $\mathrm{Lu}_{x} \mathrm{Zr}_{1-x} \mathrm{~B}_{12}$ with $0.17<x<0.8$ [30], and our work emphasizes the need to probe the intermediate doping region in order to understand the full details of the crossover.

Acknowledgments. We thank C. Topping and F. Lang for experimental assistance and M. Bristow and R. Fernandes for insightful discussions. F.K.K.K. thanks Lincoln College, Oxford, for a doctoral studentship. This study was supported by the program "Fundamental problems of high-temperature superconductivity" of the Presidium of the RAS. Part of this work was performed at the Science and Technology Facilities Council (STFC) ISIS Facility, Rutherford Appleton Laboratory, and part at $\mathrm{S} \mu \mathrm{S}$, the Swiss Muon Source (PSI, Switzerland).
[1] J. Nagamatsu, N. Nakagawa, T. Muranaka, Y. Zenitani, and J. Akimitsu, Nature (London) 410, 63 (2001).

[2] B. T. Matthias, T. H. Geballe, K. Andres, E. Corenzwit, G. W. Hull, and J. P. Maita, Science 159, 530 (1968).

[3] Y. B. Kuzma, Crystal Chemistry of Borides (Vysshaya Shkola, Lviv, Moscow, 1983).

[4] A. P. Dudka, O. N. Khrykina, N. B. Bolotina, N. Y. Shitsevalova, V. B. Filipov, and N. E. Sluchanko, J. Alloys Compd. 692, 535 (2017).

[5] I. R. Shein and A. L. Ivanovskii, Phys. Solid State 45, 1429 (2003).

[6] B. Jäger, S. Paluch, O. J. Żogał, W. Wolf, P. Herzig, V. B. Filippov, N. Shitsevalova, and Y. Paderno, J. Phys.: Condens. Matter 18, 2525 (2006).

[7] J. Teyssier, A. B. Kuzmenko, D. van der Marel, F. Marsiglio, A. B. Liashchenko, N. Shitsevalova, and V. Filippov, Phys. Rev. B 75, 134503 (2007).

[8] H. Werheit, Y. Paderno, V. Filippov, V. Paderno, A. Pietraszko, M. Armbrüster, and U. Schwarz, J. Solid State Chem. 179, 2761 (2006).

[9] J. Teyssier, R. Lortz, A. Petrovic, D. van der Marel, V. Filippov, and N. Shitsevalova, Phys. Rev. B 78, 134504 (2008).

[10] R. Lortz, Y. Wang, S. Abe, C. Meingast, Y. B. Paderno, V. Filippov, and A. Junod, Phys. Rev. B 72, 024547 (2005).

[11] A. V. Rybina, K. S. Nemkovski, P. A. Alekseev, J.-M. Mignot, E. S. Clementyev, M. Johnson, L. Capogna, A. V. Dukhnenko, A. B. Lyashenko, and V. B. Filippov, Phys. Rev. B 82, 024302 (2010)
[12] N. Sluchanko, S. Gavrilkin, K. Mitsen, A. Kuznetsov, I. Sannikov, V. Glushkov, S. Demishev, A. Azarevich, A. Bogach, A. Lyashenko, A. Dukhnenko, V. Filipov, S. Gabani, K. Flachbart, J. Vanacken, G. Zhang, and V. Moshchalkov, J. Supercond. Nov. Magn. 26, 1663 (2013).

[13] N. Sluchanko, A. Bogach, N. Bolotina, V. Glushkov, S. Demishev, A. Dudka, V. Krasnorussky, O. Khrykina, K. Krasikov, V. Mironov, V. B. Filipov, and N. Shitsevalova, Phys. Rev. B 97, 035150 (2018).

[14] K. Flachbart, S. Gabáni, K. Gloos, M. Meissner, M. Opel, Y. Paderno, V. Pavlík, P. Samuely, E. Schuberth, N. Shitsevalova, K. Siemensmeyer, and P. Szabó, J. Low Temp. Phys. 140, 339 (2005).

[15] D. Daghero, R. S. Gonnelli, G. A. Ummarino, A. Calzolari, V. Dellarocca, V. A. Stepanov, V. B. Filippov, and Y. B. Paderno, Supercond. Sci. Technol. 17, S250 (2004).

[16] M. I. Tsindlekht, G. I. Leviev, I. Asulin, A. Sharoni, O. Millo, I. Felner, Y. B. Paderno, V. B. Filippov, and M. A. Belogolovskii, Phys. Rev. B 69, 212508 (2004).

[17] V. A. Gasparov, N. S. Sidorov, and I. I. Zverkova, Phys. Rev. B 73, 094510 (2006).

[18] P. W. Anderson, Phys. Rev. Lett. 3, 325 (1959).

[19] A. A. Abrikosov and L. P. Gor'kov, Sov. Phys. JETP 12, 337 (1961).

[20] A. V. Balatsky, I. Vekhter, and J. X. Zhu, Rev. Mod. Phys. 78, 373 (2006).

[21] H. Alloul, J. Bobroff, M. Gabay, and P. J. Hirschfeld, Rev. Mod. Phys. 81, 45 (2009) 
[22] N. E. Sluchanko, A. N. Azarevich, M. A. Anisimov, A. V. Bogach, S. Y. Gavrilkin, M. I. Gilmanov, V. V. Glushkov, S. V. Demishev, A. L. Khoroshilov, A. V. Dukhnenko, K. V. Mitsen, N. Y. Shitsevalova, V. B. Filippov, V. V. Voronov, and K. Flachbart, Phys. Rev. B 93, 085130 (2016).

[23] N. Sluchanko, A. Azarevich, A. Bogach, S. Gavrilkin, V. Glushkov, S. Demishev, K. Mitsen, N. Shitsevalova, V. Filippov, S. Gabani, and K. Flachbart, Acta Phys. Polon. A 131, 1036 (2017).

[24] N. Sluchanko, V. Glushkov, S. Demishev, A. Azarevich, M. Anisimov, A. Bogach, V. Voronov, S. Gavrilkin, K. Mitsen, A. Kuznetsov, I. Sannikov, N. Shitsevalova, V. Filipov, M. Kondrin, S. Gabáni, and K. Flachbart, Phys. Rev. B 96, 144501 (2017).

[25] S. J. Blundell, Contemp. Phys. 40, 175 (1999).

[26] A. A. Yaouanc and P. D. de. Réotier, in Muon Spin Rotation, Relaxation, and Resonance: Applications to Condensed Matter (Oxford University Press, Oxford, UK, 2011), p. 486.

[27] P. J. C. King, R. de Renzi, S. P. Cottrell, A. D. Hillier, and S. F. J. Cox, Phys. Scr. 88, 068502 (2013).

[28] F. L. Pratt, Physica B: Condens. Matter 289-290, 710 (2000).

[29] See Supplemental Material at http://link.aps.org/supplemental/ 10.1103/PhysRevB.98.094505 for further discussion of the nodal and gapped fitting and additional plots of the superconducting parameters.

[30] N. E. Sluchanko et al. (unpublished).

[31] E. H. Brandt, Phys. Rev. B 68, 054506 (2003).

[32] B. S. Chandrasekhar and D. Einzel, Ann. Phys. (Berl.) 505, 535 (1993).
[33] M. J. Graf, S. K. Yip, J. A. Sauls, and D. Rainer, Phys. Rev. B 53, 15147 (1996).

[34] J.-P. Reid, A. Juneau-Fecteau, R. T. Gordon, S. René de Cotret, N. Doiron-Leyraud, X. G. Luo, H. Shakeripour, J. Chang, M. A. Tanatar, H. Kim, R. Prozorov, T. Saito, H. Fukazawa, Y. Kohori, K. Kihou, C. H. Lee, A. Iyo, H. Eisaki, B. Shen, H.-H. Wen, and L. Taillefer, Supercond. Sci. Technol. 25, 084013 (2012).

[35] Z. Guguchia, A. Amato, J. Kang, H. Luetkens, P. K. Biswas, G. Prando, F. von Rohr, Z. Bukowski, A. Shengelaya, H. Keller, E. Morenzoni, R. Fernandes, and R. Khasanov, Nat. Commun. 6, 8863 (2015).

[36] R. Kubo and T. Toyabe, in Magnetic Resonance and Relaxation, edited by R. Blinc (North-Holland, Amsterdam, 1967), p. 810.

[37] R. S. Hayano, Y. J. Uemura, J. Imazato, N. Nishida, T. Yamazaki, and R. Kubo, Phys. Rev. B 20, 850 (1979).

[38] D. M. Maric, P. F. Meier, S. Vogel, S. F. J. Cox, and E. A. Davis, J. Phys.: Condens. Matter 3, 9675 (1991).

[39] S. F. J. Cox, Z. Naturforsch. A 47, 371 (1992).

[40] J. S. Möller, P. Bonfà, D. Ceresoli, F. Bernardini, S. J. Blundell, T. Lancaster, R. De Renzi, N. Marzari, I. Watanabe, S. Sulaiman, and M. I. Mohamed-Ibrahim, Phys. Scr. 88, 068510 (2013).

[41] F. R. Foronda, F. Lang, J. S. Möller, T. Lancaster, A. T. Boothroyd, F. L. Pratt, S. R. Giblin, D. Prabhakaran, and S. J. Blundell, Phys. Rev. Lett. 114, 017602 (2015).

[42] R. E. Walstedt and L. R. Walker, Phys. Rev. B 9, 4857 (1974).

[43] G. Kalvius, D. Noakes, N. Marcano, R. Wäppling, F. Iga, and T. Takabatake, Physica B 326, 398 (2003). 